\title{
Excitation and temperature dependence of the broad gain spectrum in GaAs/AIGaAs quantum rings
}

\author{
Juyeong Jang, ${ }^{1}$ Seunghwan Lee, ${ }^{1, a)}$ Minju Kim, ${ }^{1,}$ a) Sunwoo Woo, ${ }^{1}$ Inhong Kim, ${ }^{1}$ Jihoon \\ Kyhm, ${ }^{2}$ Jindong Song, ${ }^{3}$ Robert A. Taylor, ${ }^{4}$ and Kwangseuk Kyhm ${ }^{1, b}$ ) \\ 1) Department of Opto-mechatronics and Cogno-mechatronics Engineering, \\ RCDAMP, Pusan National University, Busan 46241, Republic of Korea \\ 2) Quantum Functional Semiconductor Research Center, Donguk University, Seoul, \\ 04620, Republic of Korea \\ 3) Nano-Photonics Research Center, KIST, Seoul, 136-791, \\ Republic of Korea \\ 4) Clarendon Laboratory, Department of Physics, University of Oxford, \\ Oxford OX1 3PU, UK
}

(Dated: 8 October 2020)

We have employed a variable stripe length method in order to measure the optical gain of GaAs/AlGaAs quantum rings. Although the large lateral diameter of quantum rings $(\sim 50 \mathrm{~nm})$ with a few $\mathrm{nm}$ size distribution is expected to cause a small spectral inhomogeneity $(\sim 1 \%)$, a broad gain width $(\sim 300 \mathrm{meV})$ was observed. This result was attributed to a variation of the vertical heights and variations in localized states that exhibit crescent shaped wavefunctions, whereby the energy levels are distributed over a broad spectral range. When the excitation intensity is decreased, irregular peaks appear in the gain spectrum gradually. Similar phenomena were also observed with increased temperature. We conclude that excited carriers in quantum rings are distributed stochastically at various localized states, and the population inversion is sensitive to excitation intensity and temperature.

\footnotetext{
a) equally contributed to the first author

b)E-mail: kskyhm@pusan.ac.kr
} 
Recently, the droplet method has enabled the growth of semiconductor quantum rings $(\mathrm{QRs})^{1}$, where an orbital angular momentum around the ring is given to electrons and holes. When quantum coherence is present in a $\mathrm{QR}$, the rotational motion around the rim shows a novel quantum nature. With an increasing external magnetic field $(B)$ threading the lateral cross section of QRs, the Aharonov-Bohm effect can be observed in both optical ${ }^{2}$ and electrical $^{3}$ experiments. Regarding the lateral image of QRs, the rim confinement is often simplified by a one-dimensional loop model. However, the morphology of QRs is known to be volcano-like $e^{4,5}$. The rim width is not small $(\sim 20 \mathrm{~nm})$ enough to be ignored, and the rim height is anisotropic. As a result, the lateral wavefunction of QRs is likely to be localized with a crescent-like shape.

It is also noticeable that the lateral diameter of droplet quantum structures is large $(\sim 50 \mathrm{~nm})$ compared to those of Stranski-Krastanov (SK) quantum dots (QDs) $(\sim 20 \mathrm{~nm})$ and colloidal quantum dots $(\sim 5 \mathrm{~nm})$. Provided that only a few nanometer variation occurs around the average lateral size of QRs, the spectral inhomogeneity of QRs is expected to be less than $\sim 1 \%$, which is smaller by an order of magnitude than that of both SK and colloidal quantum dots. Nevertheless, ensemble QRs show a broad emission spectrum ${ }^{1}$. Therefore, the spectral inhomogeneity of QRs is dominated by a variation of the vertical height rather than the lateral size. Recently, it was shown that various excited states are present in a single $\mathrm{QR}^{1,4}$, but the wavefunctions are strongly localized ${ }^{6}$. Even with weak excitation, the localized states are likely to be observed at high energies in the emission spectrum $^{6,7}$. Therefore, the broad spectrum in QRs originates from the various localized states, and their energy levels are finely spaced.

Recently, the broad spectrum of SK QDs was already used for multi-wavelength laser, semiconductor optical amplifier, and short pulse laser applications ${ }^{8-10}$. While SK QD lasers have low threshold current densities $\left(J_{\text {th }} \sim 10 \mathrm{Acm}^{-2}\right)$, their temperature instability still needs to be resolved. For example, the threshold current density of SK QDs still shows a temperature dependence, arising from a non-equilibrium state of the inhomogeneous QDs ${ }^{11,12}$. Given the energy distribution of separate SK QDs, the carrier distribution is mediated via thermal carriers in the wetting layers. Provided that the activation temperature is not too high, a non-equilibrium state is likely to occur in the inhomogeneous QDs, resulting in an increased threshold density of lasing. On the other hand, the equilibrium state of QRs is achieved through intra-relaxation due to the absence of a wetting layer and the large dis- 
tance between QRs. Therefore, a broad gain spectrum can be obtained from a low density of QRs, and light amplification in a single QR is also possible. The droplet epitaxy of QRs is also advantageous in controlling the spectral range. The size control of SK QDs is limited when strain-induced growth conditions are considered, but droplet epitaxy enables the control of the size and morphology of QRs without a wetting layer. In this work, we studied optical gain in QRs in order to consider the possibility of laser applications. In particular, we have investigated excitation and temperature dependence of the optical gain spectrum and studied the nature of localized states.

In Fig. 1(a), the adiabatic potentials are plotted schematically as a function of radius $(\rho)$ for a QR structure obtained by atomic force microscopy (AFM) (Fig. 1(b)). Because the rim height $(<10 \mathrm{~nm})$ is small compared with the lateral size $(\sim 50 \mathrm{~nm})$, the lateral wavefunction of a QR can be considered as slowly varying for variations in the height of the rings. In this case, the vertical states can be separated from the lateral states through the adiabatic approximation ${ }^{1,5}$. As a result, an adiabatic potential valley $\left(\varepsilon^{k}(\rho, \phi)\right)$ for a vertical quantum state $k$ can be obtained as a function of azimuthal angle $\phi^{4,6}$. For example, when the excited vertical confinement state of $k=2$ is considered, the radial dependence of $\varepsilon^{k=2}(\rho, \phi)$ becomes different for varying $\phi$ due to the anisotropic structure of the QRs, i.e. the energy depth of $\varepsilon^{k=2}(\rho,|\phi|>0)$ is shallow compared to that of $\varepsilon^{k=2}(\rho, \phi=0)$. As a result, the eigenstates determined by the anisotropic $\varepsilon^{k}(\rho, \phi)$ are localized with a crescent-like shape.

The shape of $\varepsilon^{k}(\rho, \phi)$ leads to lateral confinement states that are separated by several $\mathrm{meV}$. When the two separate crescent-like localized states merge through the tunneling effect, the quantized rotational motion can become involved with sub meV level spacing. Consequently, these fine levels give rise to a quasi-continuous spectrum leading to a linewidth broadening effect. Fig. 1(c) shows a scanning electron microscope (SEM) image of uncapped GaAs QRs grown by the droplet method. The lateral shape of the QRs looks elliptical with a major diameter of $\sim 50 \mathrm{~nm}$, and the density of QRs is $1.6 \times 10^{10} \mathrm{~cm}^{-2}$. We also obtained the average surface-to-surface distance between QRs as $\sim 51 \mathrm{~nm}$. In this case, the separation of QRs is large enough to rule out a direct exciton hopping through the dipole-dipole interaction.

For a confinement energy level $E_{n}^{k}$ determined by the adiabatic potential (Fig.1(a)), the inhomogeneous linewidth $\left(\delta E_{n}^{k}\right)$ can be given by $\delta E_{n}^{k} \sim \delta \rho_{\mathrm{w}} /<\rho_{\mathrm{w}}>^{3}$, where $\rho_{\mathrm{w}}$ and $<\rho_{\mathrm{w}}>$ 


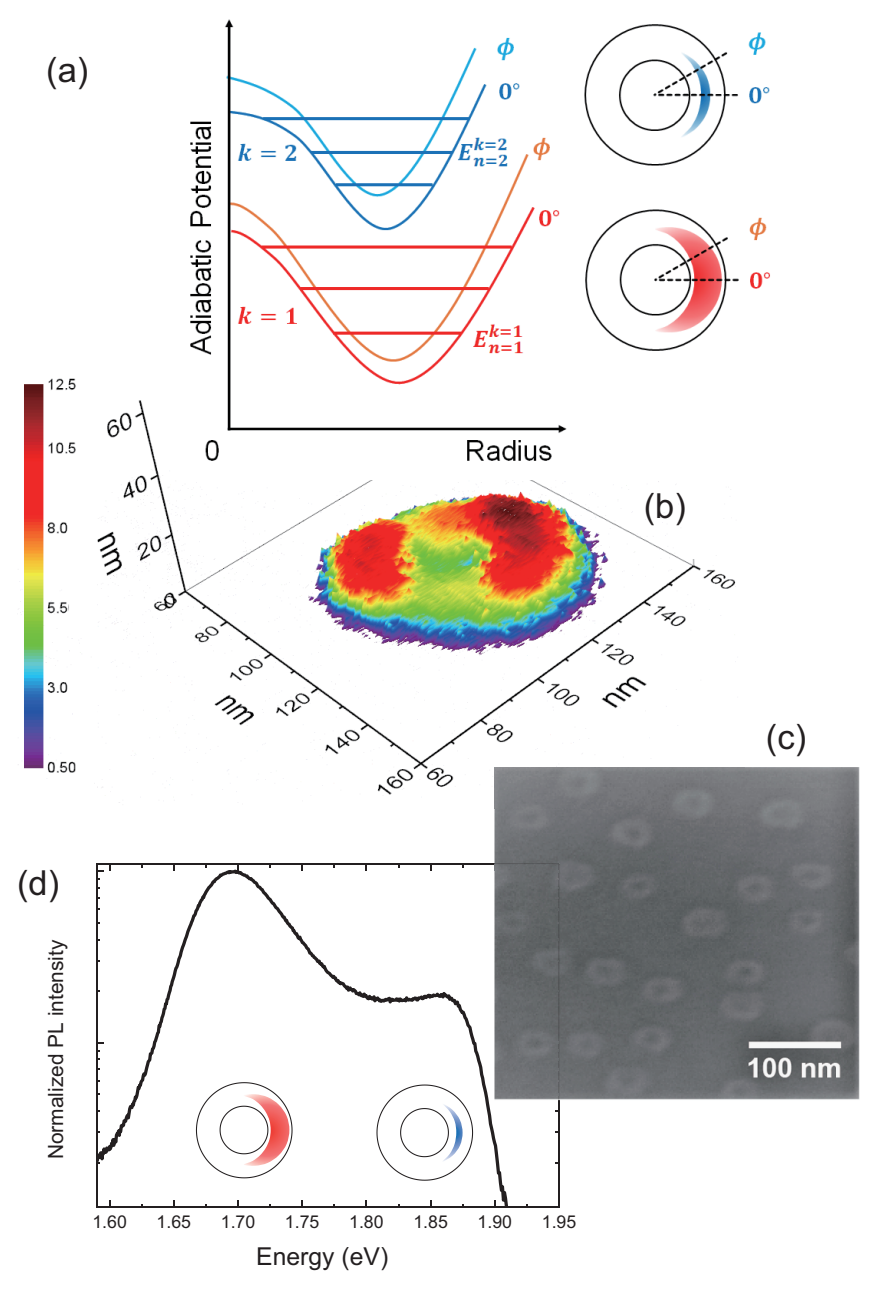

FIG. 1. The adiabatic potentials of two different vertical quantum states $(k=1,2)$ are shown schematically as a function of radius and azimuthal angle $(\phi)(\mathrm{a})$, The anisotropic structure of QRs obtained by AFM (b) and SEM (c) images of uncapped GaAs quantum rings. (d) GaAs/AlGaAs quantum rings show a broad PL spectrum due to the various crescent-like localized states.

are a variation and an average of the lateral confinement width. Although the lateral diameter of QRs barely changes, the height variation of QRs gives rise to a significant change of $\rho_{\mathrm{w}}$ through modification of the adiabatic potential. In Fig. 1(d), GaAs/AlGaAs QRs at $4 \mathrm{~K}$ show a broad photoluminescence (PL) spectrum, where the fine confinement levels determined by the adiabatic potentials are distributed in between bulk GaAs and barrier AlGaAs. The various fine levels $\left(E_{n}^{k}\right)$ of a single $\mathrm{QR}$ are separated by several meV, and coexist in the same $\mathrm{QR}^{4}$. Therefore, the spectral inhomogeneity of $\mathrm{QRs}$ is dominated by height variation. 

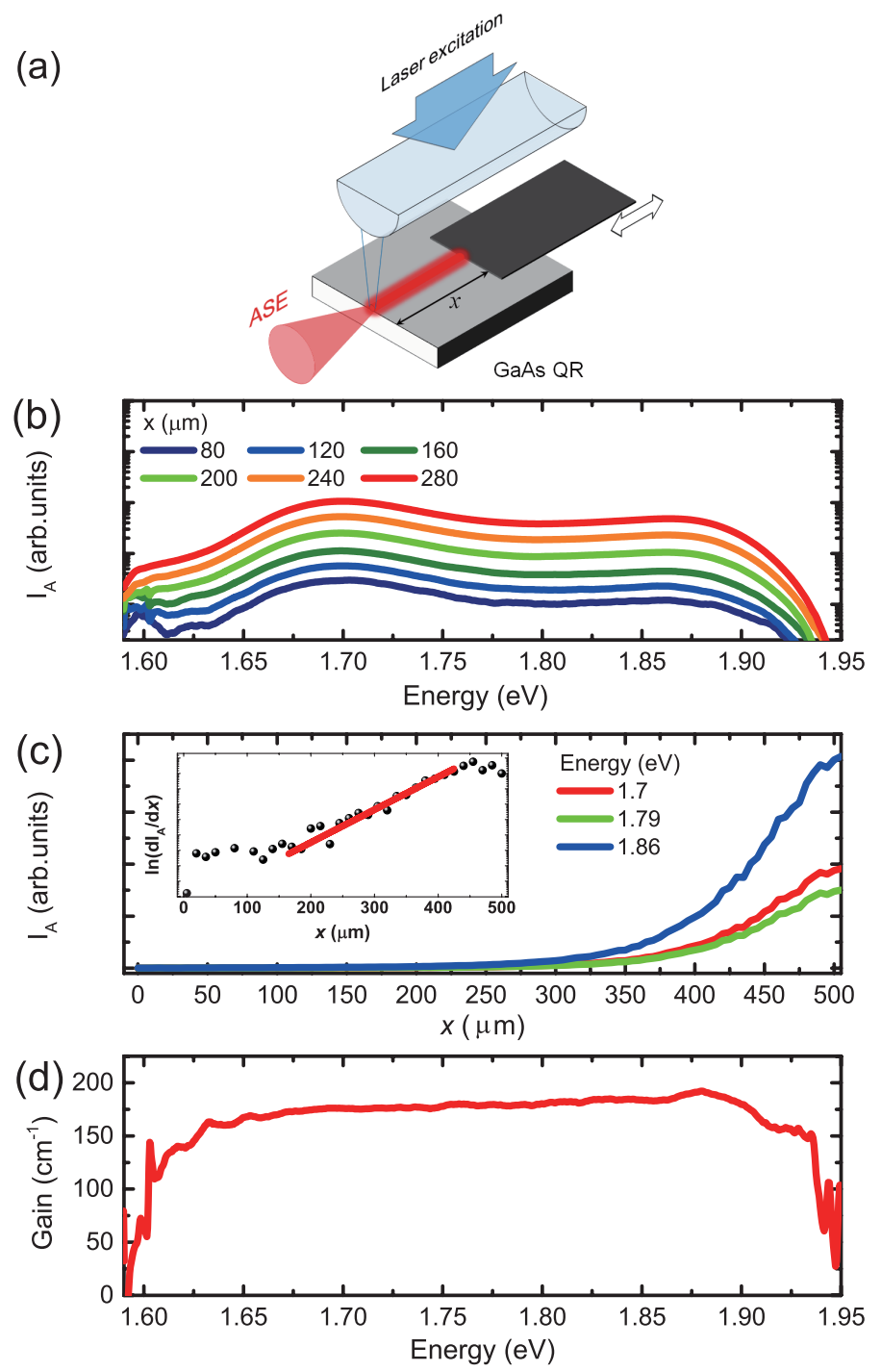

FIG. 2. (a) A schematic of the variable stripe length method for optical gain measurement. (b) With increasing stripe length $(x)$, the edge emission spectrum of amplified spontaneous emission $\left(I_{\mathrm{A}}\right)$ is measured at $4 \mathrm{~K}$. (c) Stripe length dependent $I_{\mathrm{A}}(x)$ is plotted at various emission energies, where the inset shows the slope on a $\log$ scale $\left(\ln \left(\mathrm{d} I_{\mathrm{A}} / \mathrm{d} x\right) \sim g x\right)$. (d) Gain spectrum of quantum rings at $4 \mathrm{~K}$ under $3.1 \mathrm{kWcm}^{-2}$ excitation.

In order to examine light amplification in the broad spectrum of QRs, a variable stripe length method (VSLM) was used to obtain optical gain as shown in Fig. 2(a). For excitation, pulsed light $(\sim 200 \mathrm{fs})$ from a Ti:sapphire laser operating at an $80 \mathrm{MHz}$ repetition rate was frequency-doubled $(\sim 400 \mathrm{~nm})$, and a beam stripe was generated by a cylindrical lens. Given an extended beam stripe, we selected a nearly uniform intensity range. As light am- 
plification proceeds along the excited stripe, the edge emission shows an exponential growth with increasing stripe length $(x)$. A beam block was also used to control the stripe length, and the sample was mounted in a closed-recycled cryostat. With $3.1 \mathrm{kWcm}^{-2}$ excitation, the edge emission spectrum of amplified spontaneous emission $\left(I_{\mathrm{A}}\right)$ at $4 \mathrm{~K}$ were measured for various stripe lengths (Fig. 2(b)), where $I_{\mathrm{A}}$ is shown in a log scale to clarify the broad spectral width.

In Fig. 2(c), stripe length dependence of $I_{\mathrm{A}}(x)$ is shown at selected emission energies $(\hbar \omega)$. If the stipe is assumed to act as a one-dimensional waveguide, the light amplification can be described as ${ }^{13}$

$$
\frac{\mathrm{d} I_{\mathrm{A}}(\hbar \omega, x)}{\mathrm{d} x}=g(\hbar \omega) \cdot I_{\mathrm{A}}(\hbar \omega, x)+J_{\text {spon }} \Omega,
$$

where $g(\hbar \omega), J_{\text {spon }}$, and $\Omega$ are optical gain coefficient, spontaneous emission density, and solid angle of the edge emission, respectively. When the gain saturation effect is ignored, the gain coefficient becomes independent of stripe length. In this case, a solution of Eq. (1) is given by

$$
I_{\mathrm{A}}(\hbar \omega, x)=\frac{J_{\text {spon }} \Omega}{g(\hbar \omega)}\left(\mathrm{e}^{g(\hbar \omega) x}-1\right) .
$$

As shown in inset of Fig. 2(c), it should be noted that we selected a particular range of stripe length $(200 \sim 450 \mu \mathrm{m})$ to obtain an optical gain coefficient, where $\mathrm{d} I_{\mathrm{A}} / \mathrm{d} x \sim \mathrm{e}^{g x}$ is valid. While the wave guiding along the stripe is inefficient with short stripe lengths $(<200 \mu \mathrm{m})$, a gain saturation effect also occurs with long stripe lengths $(>450 \mu \mathrm{m})^{14}$. Consequently, a gain coefficient $g(\hbar \omega)$ at a certain emission energy $(\hbar \omega)$ can be obtained by considering the slope of $\ln \left(\mathrm{d} I_{\mathrm{A}} / \mathrm{d} x\right) \sim g x$ in the limited range of stripe lengths $(200 \sim 450 \mu \mathrm{m})$.

As shown in Fig. 2(d), we observed a very broad optical gain spectrum from QRs at $4 \mathrm{~K}$. In the case of SK QDs, such as those made form InAs and InGaAs, the spectral width ( $\lesssim 50 \mathrm{meV}$ ) originates from the ground and excited states with a large size distribution of the QDs. However, the broad spectral width $(\sim 300 \mathrm{meV})$ of QRs can be attributed to the fine levels $\left(E_{n}^{k}\right)$ of the two vertical confinement states $(k=1$ and $k=2)$ with a height variation. Additionally, the fine levels are localized with crescent shaped wavefunctions. Therefore, intra-relaxation between the fine levels is possibly suppressed in a similar manner to meta stable states, although they coexist in the same QR. In this case, the population inversion of the localized states is distributed stochastically over a wide energy range, and a fluctuation can be seen in the gain spectrum unless the excitation is strong enough. In order to 

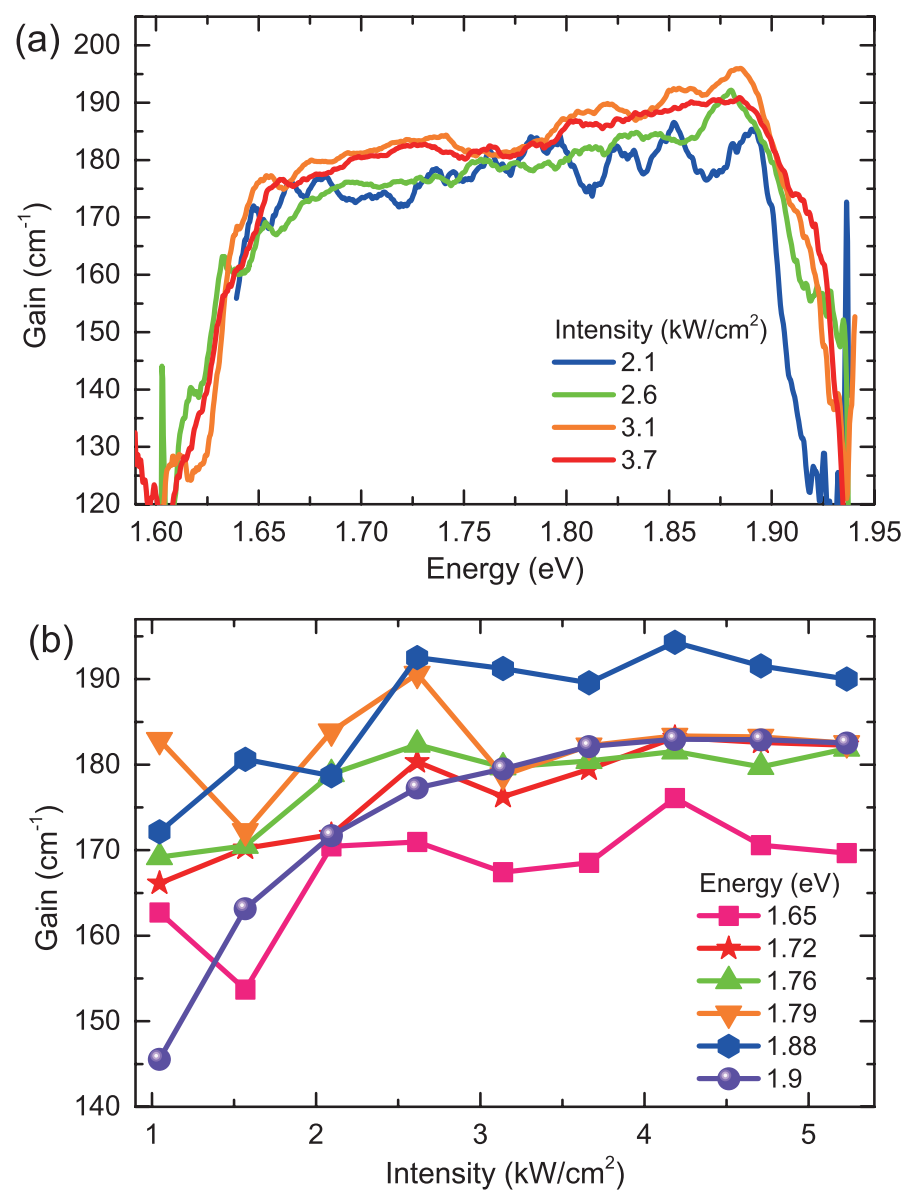

FIG. 3. Gain spectra were obtained at $4 \mathrm{~K}$ for increasing excitation intensity (a), whereby the excitation dependent gain coefficients at different emission energies were also obtained (b).

resolve this issue, we have also investigated the excitation intensity dependence of the gain spectrum at $4 \mathrm{~K}$. As the excitation intensity decreases, we found a significant fluctuation in the gain spectrum in Fig. 3(a). In Fig. 3(b), the excitation intensity dependence of the gain coefficient was also plotted at various emission energies. A high emission energy shows a drastic decrease in the gain coefficient for decreased excitation intensity. However, the gain coefficients at low emission energies look less vulnerable to decreased excitation intensity, but the gain fluctuation is significant. These results support the assertion that the intra-relaxation of the localized states in a quantum ring is interrupted. On the other hand, with increasing excitation, the carrier population of the fine states becomes saturated. As a result, the activated intra-relaxation smoothes out the irregular spikes in the gain spectrum.

We have also investigated temperature dependence of the gain spectra in $\mathrm{QRs}^{15}$. Using the measured temperature dependence of the amplified spontaneous emission in quantum 

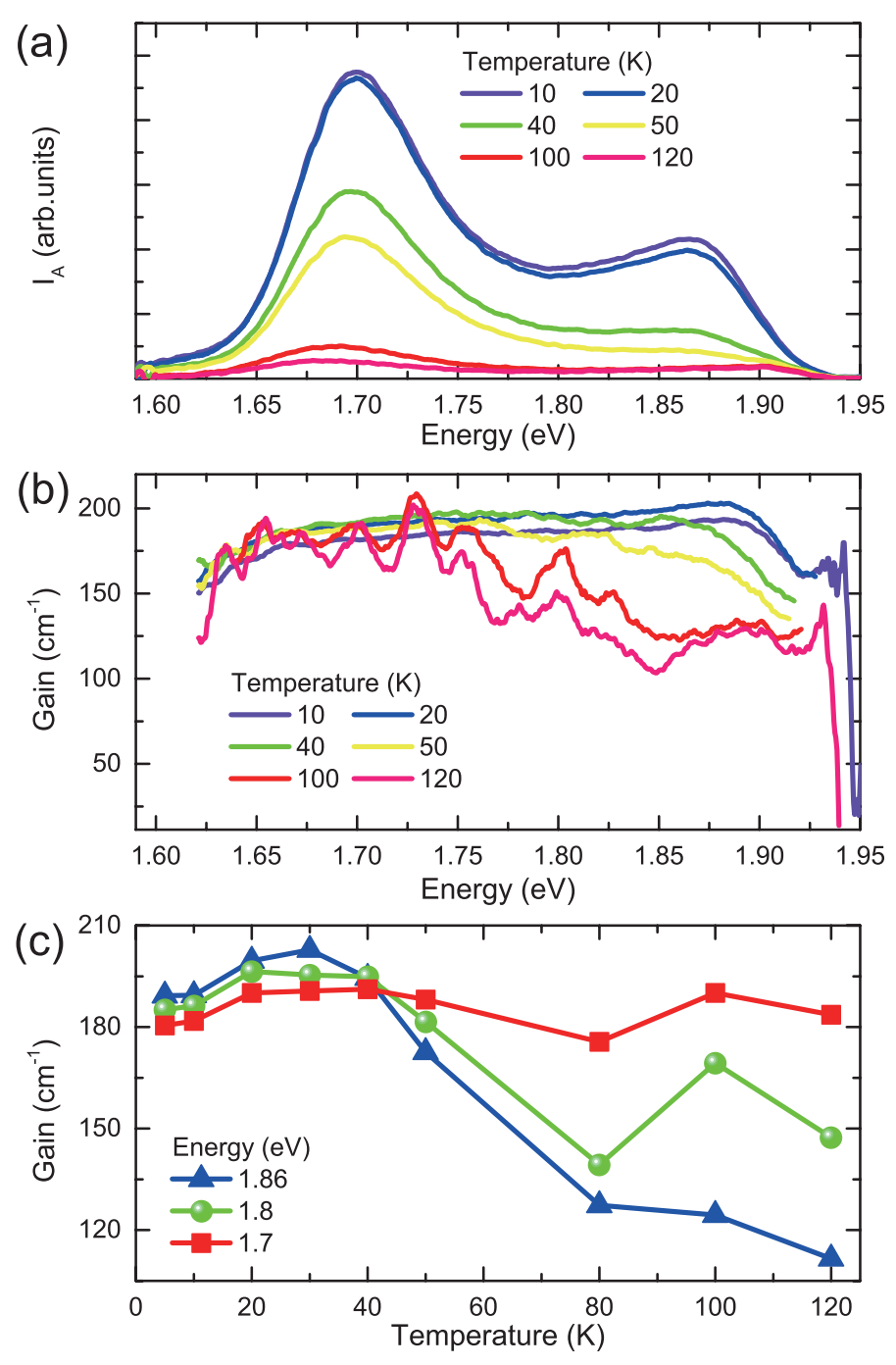

FIG. 4. Amplified spontaneous emission specta (a) and gain spectra (b) for temperature. (c) Temperature dependence of the gain coefficient is plotted at various emission energies, which are excited by the same intensity of $3.7 \mathrm{kWcm}^{-2}$.

rings shown in (Fig. 4(a)), we obtained gain spectra at various temperatures as shown in Fig. 4(b). Up to $\sim 50 \mathrm{~K}$, smooth gain spectra were seen. However, irregular peaks become significant in the gain spectrum at $100 \mathrm{~K}$ and $120 \mathrm{~K}$ with suppressed gain coefficients at high energy $(>1.77 \mathrm{eV})$. In Fig. 4(c), the gain coefficients at different emission energies were also plotted with increasing temperature. For example, the gain coefficient at $1.86 \mathrm{eV}$ decreases significantly near $\sim 80 \mathrm{~K}$. This result can be explained by thermal excitation. Although the fine levels in the high-energy spectra are also localized states, the depth of the adiabatic potential of $\left.\varepsilon^{k=2}(\rho, \phi)\right)$ is shallow. Therefore, the localized carriers in the high energy lev- 
els become unstable due to thermal activation as the temperature increases, resulting in a decreased population inversion for the high energy spectra compared to that for the low energy spectra. Nevertheless, the gain for the low energy spectra $(\lesssim 1.77 \mathrm{eV})$ looks less susceptible to temperature increase, although the irregular spikes in the gain spectra become significant. This result can be explained by thermal activation of trapped states. Although GaAs/AlGaAs QRs have a strain free structure compared to InAs and InGaAs QDs, carriers can be trapped at the interface between the QRs and the AlGaAs barrier due to defects. Increasing the temperature activates the trapped states. As a result, the emission at the low energy end of the spectra becomes dominant while the high energy component is suppressed (Fig. 4(a)). As shown in Fig. 4(c), the gain coefficients at different emission energy are maximized near $\sim 30 \mathrm{~K}$, and this result can also be explained by the thermal activation of trapped states.

For practical laser applications, the gain can be increased by increasing the number of stacked QD layers, where the optical loss increase saturates, and the temperature dependent gain can be used for switchable multi-wavelenth lasers. Recently, lasing operation was demonstrated using stacked layers of InGaAs/GaAs QRs ${ }^{16}$. With a $1 \mathrm{~mm}$ cavity length, stimulated emission was observed up to $150 \mathrm{~K}$. Interestingly, they also found that $J_{\text {th }}$ increases when the temperature falls below $77 \mathrm{~K}$. This result also supports the presence of trapped states in QRs. While InGaAs/GaAs QRs provide near-IR lasing (900 $950 \mathrm{~nm}$ ), GaAs/AlGaAs QRs can cover the red end of the visible range $(670 \sim 740 \mathrm{~nm})$.

In summary, we found a broad gain spectrum is present in quantum rings with a $\mathrm{QR}$ density of $1.6 \times 10^{10} \mathrm{~cm}^{-2}$. The broad spectral width is dominated by the vertical height variation and the fine energy levels of localized states, which are distributed over a wide spectral energy. Due to the nature of localized states, the fine levels seem to be occupied in a stochastic manner, and the intra-relaxation becomes suppressed at low temperature. Consequently, irregular peaks appear in the gain spectrum unless either the excitation intensity or the thermal energy is large enough.

\section{ACKNOWLEDGMENTS}

This work was supported by Basic Science Research Program (NRF-2020R1A2C1005973). 


\section{REFERENCES}

${ }^{1}$ V. Fomin, Physics of Quantum rings (2nd Edition, Springer International Publishing AG, Switzerland, 2018).

${ }^{2}$ H. Kim, S. Park, R. Okuyama, K. Kyhm, M. Eto, R. Taylor, G. Nogues, L. Dang, M. Potemski, K. Je, J. Kim, J. Kyhm, and J. Song, Nano Letters 18, 6188 (2018).

${ }^{3}$ A. Lorke, R. J. Luyken, A. O. Govorov, J. P. Kotthaus, J. M. Garcia, and P. M. Petroff, Phys. Rev. Lett., 84, 2223 (2000).

${ }^{4}$ H. Kim, W. Lee, S. Park, K. Kyhm, K. Je, R. Taylor, G. Nogues, L. Dang, and J. Song, Sci. Rep., 7, 40026 (2017).

${ }^{5}$ N. A. J. M. Kleemans, I. M. A. Bominaar-Silkens, V. M. Fomin, V. N. Gladilin, D. Granados, A. G. Taboada, J. M. Garcia, P. Offermans, U. Zeitler, P. C. M. Charistianen, J. C. Maan, J. T. Devreese, and P. M. Koenraad, Phys. Rev. Lett., 99, 146808 (2007).

${ }^{6}$ H. D. Kim, K. Kyhm, R. A. Taylor, G. Nogues, K. C. Je, E. H. Lee, and J. D. Song, Appl. Phys. Lett. 102, 033112 (2013).

${ }^{7}$ H. D. Kim, K. Kyhm, R. A. Taylor, A. A. L. Nicolet, M. Potemski, G. Nogues, K. C. Je, E. H. Lee, and J. D. Song, Appl. Phys. Lett. 103, 173106 (2013).

${ }^{8}$ E. U. Rafailov, M. A. Cataluna, and W. Sibbett, Nat. Photonics 1, 395 (2007).

${ }^{9}$ D. L. Huffaker, G. Park, Z. Zou, O. B. Shchekin, and D. G. Deppe, Appl. Phys. Lett. 73, 2564 (2019).

${ }^{10}$ E. U. Rafailov, M. A. Cataluna, W. Sibbett, N. D. Il'inskaya, Y. M. Zadiranov, A. E. Zhukov, V. M. Ustinov, D. A. Livshits, A. R. Kovsh, and N. N. Ledentsov, Appl. Phys. Lett. 87, 081107 (2005).

${ }^{11}$ H. D. Summers, J. D. Thomson, P. M. Smowton, P. Blood, and M. Hopkinson, Semicond. Sci. Technol. 16, 140(2001).

${ }^{12}$ M. Hutchings, I. O'Driscoll, P. M. Smowton, and P. Blood, Appl. Phys. Lett. 104, 031103(2014).

${ }^{13}$ J. Jang, G. Song, K. Kyhm, and C. Cho, Appl. Phys. Lett. 114, 081101 (2019).

${ }^{14}$ K. Kyhm, R. A. Taylor, and J. F. Ryan, Appl. Phys. Lett. 79, 3434 (2001).

${ }^{15}$ G. Park, O. B. Shchekin, and D. G. Deppe, IEEE J. Quantum Electron. 36, 1065 (2000).

${ }^{16}$ F. Suarez, D. Granados, M. Luisa Doctor, and J. M. Garcia, Nanotechnology 15, S126(2004). 